\title{
Abstract
}

\section{On Symmetry Restoration in General Relativity ${ }^{\dagger}$}

\author{
Luciano Rezzolla \\ Institute for Theoretical Physics, Goethe University Frankfurt, 60438 Frankfurt, Germany; \\ rezzolla@itp.uni-frankfurt.de \\ + Presented at Symmetry 2017-The First International Conference on Symmetry, Barcelona, Spain, \\ 16-18 October 2017.
}

Published: 12 January 2018

Einstein's theory of general relativity is often regarded as the best theory of gravity that we know. Yet, this theory often manifests itself under conditions where no symmetry is present and nonlinear dynamics dominates. I will discuss how these conditions are systematically accompanied by the restoration of some degree of symmetry. Hence, despite gravity appearing often under conditions devoid of symmetry, asymptotic solutions tend to restore symmetry.

(C) 2018 by the author. Licensee MDPI, Basel, Switzerland. This article is an open access article distributed under the terms and conditions of the Creative Commons Attribution (CC BY) license (http://creativecommons.org/licenses/by/4.0/). 